\title{
Polimorfismos en los genes de desintoxicación CYP1A1, CYP2E1, GSTT1 y GSTM1 en la susceptibilidad al cáncer gástrico
}

\author{
Auxiliadora González ${ }^{1 *}$, Vanessa Ramírez ${ }^{1,3}$, Patricia Cuenca ${ }^{1,2}$ \& Rafaela Sierra 1,3 \\ 1 Instituto de Investigaciones en Salud (INISA). Universidad de Costa Rica, 2060, San José, Costa Rica. \\ 2 Escuela de Biología. Universidad de Costa Rica, 2060, San José, Costa Rica. \\ 3 Escuela de Nutrición. Universidad de Costa Rica, 2060, San José, Costa Rica. \\ * Fax: (506) 207 5130; auxigv@ costarricense.cr
}

Recibido 06-IX-2003. C Corregido 31-II-2004. Aceptado 09-II-2004.

\begin{abstract}
Polymorphisms in detoxification genes CYP1A1, CYP2E1, GSTT1 and GSTM1 in gastric cancer susceptibility. Cytochrome P450 (CYP) and glutathione S-transferase (GST) enzymes are involved in activation and detoxification of many potential carcinogens. Genetic polymorphisms in those enzymes have been found to influence the interindividual susceptibility to cancer. Some polymorphisms of those enzymes have been associated specifically with susceptibility to gastric cancer. We conducted a study in a Costa Rican population, where gastric cancer incidence and mortality rates are among the highest in the world. We investigated whether such variations affected the risk of developing gastric cancer. Subjects included 31 with gastric cancer, 58 controls with gastric injures others than cancer and 51 normal controls confirmed by X-rays (double-contrast) or endoscopic diagnostic. DNA from peripheral white blood cell was obtained from all subjects. Deletion of GSTT1 and GSTM1 was assessed by multiplex PCR and genotyping of CYP2E1 was performed using a PCRbased restriction fragment length polymorphism assay with the restriction enzyme PstI and the gene CYP1A1 using the restriction enzyme MspI. The prevalence of CYP1A1 MspI polymorphism, GSTT1 and GSTM1 null genotype was similar in the three groups of individuals $(\mathrm{p}=0.73, \mathrm{p}=0.88 \mathrm{y} \mathrm{p}=0.89$ respectively). Our findings suggest that the polymorphism CYP2E1 PstI could be associated with a reduced risk of having gastric cancer $\left(\mathrm{OR}=0.09, \mathrm{IC}_{95 \%}: 0.01\right.$ - 0.83). Rev. Biol. Trop. 52(3): 591-600. Epub 2004 Dic 15.
\end{abstract}

Key words: gastric cancer, genetic susceptibility, molecular epidemiology, cytochrome P450 (CYP), glutathione S-transferase, (GST).

Palabras clave: cáncer gástrico, susceptibilidad genética, epidemiología molecular, citocromo P450 (CYP), glutation S-transferasa, (GST).

El cáncer gástrico es la segunda causa de muerte por cáncer en el mundo (Parkin y Pisani 1999). Es responsable de casi un millón de nuevos casos anualmente (Ferlay et al. 2001). Costa Rica es uno de los países con mayores tasas de incidencia y mortalidad por este cáncer (Sierra et al. 1995, Ferlay et al. 2001).

Los tipos más frecuentes de cáncer gástrico son el tipo intestinal y el tipo difuso. Ambos difieren en su etiología, epidemiología y mecanismos biológicos. El tipo intestinal presenta una mayor incidencia, es más frecuente en hombres que en mujeres y se ha relacionado con factores ambientales, mientras que el difuso, es menos frecuente, afecta por igual a individuos de ambos sexos y está relacionado con factores genéticos (Shibata et al. 2001).

El desarrollo del cáncer gástrico de tipo intestinal, es un proceso con varios estadios entre los cuales intervienen múltiples factores de riesgo genéticos y ambientales que facilitan, retrasan o evitan su aparición. Los cambios que preceden a este tipo de cáncer son la gastritis crónica atrófica, la metaplasia y la displasia (Correa 1992). Algunos de los factores ambientales asociados con el incremento 
del riesgo a desarrollar este tipo de cáncer son el bajo nivel socioeconómico, la producción endógena de nitrosaminas, la infección por $\mathrm{He}$ licobacter pylori, la ingesta de productos con alto contenido de sal o productos nitrosados e irritantes, el bajo consumo de frutas y verduras y la historia familiar de cáncer gástrico (Anónimo 1997, Sierra 2002).

Cada individuo tiene un grado diferente de susceptibilidad de llegar a desarrollar cáncer gástrico. Estas diferencias pueden ser producto de ciertos polimorfismos genéticos. Algunos de los polimorfismos que han sido estudiados como factores de riesgo de cáncer gástrico son los polimorfismos del gen de la interleucina 1- $\beta$ (El-Omar et al. 2000) o de genes involucrados en el metabolismo de sustancias cancerígenas, entre otros (Hengstler et al. 1998, Nishimoto et al. 2000, Cai et al. 2001, Saadat y Saadat 2001).

Actualmente las principales enzimas conocidas involucradas en el metabolismo de sustancias cancerígenas pertenecen a las familias citrocromo $\mathrm{P}_{450}$ y Glutatión S-transferasa. Estas participan en la activación y desintoxicación de sustancias que podrían actuar como factores de riesgo o de protección de cáncer gástrico. Las sustancias cancerígenas pueden provenir de los alimentos, drogas u otro tipo de sustancias químicas que ingresan al cuerpo o de sustancias endógenas (Lang y Pelkonen 1999). Los individuos que cargan la forma más activa de una enzima relacionada con la activación de los carcinógenos, o los alelos menos eficientes de las enzimas de desintoxicación, podrían presentar un mayor riesgo de desarrollar cáncer (Krajinovic et al. 1999).

En la presente investigación se estudió la asociación entre las lesiones gástricas leves (tipo I y II) y el cáncer gástrico, con los polimorfismos de desintoxicación química CYP1A1 MspI y CYP2E1 Pst I, de mayor expresión enzimática y los polimorfismos de los genes GSTM1 y GSTT1 que carecen de un producto proteínico funcional, en una población costarricense de alto riesgo para cáncer gástrico.

\section{MATERIALES Y MÉTODOS}

Población de estudio: La muestra del presente estudio fue seleccionada a partir de la población que participó en una investigación más amplia sobre: Marcadores biológicos en sangre para la identificación de personas con alto riesgo de cáncer gástrico, que se llevó a cabo con personas que acudían al Centro de Detección Temprana de Cáncer Gástrico del Hospital Max Peralta y con pacientes del Hospital Calderón Guardia.

Se seleccionaron 140 individuos con edades entre los 18 y 93 años y se clasificaron en tres grupos. Un primer grupo está formado por 51 personas sin sospecha de cáncer gástrico (grupo control), según el examen por rayos $\mathrm{X}$ (serie gastroduodenal de doble contraste) que se llevó a cabo con equipos de alta resolución siguiendo el método Japonés del Centro de Detección Temprana de Cáncer Gástrico o sin cáncer gástrico según el diagnóstico endoscópico de ambos hospitales. Un segundo grupo conformado por 58 pacientes con lesiones gástricas leves. El tercer grupo corresponde a 31 individuos diagnosticados con cáncer gástrico por patólogos especialistas.

Análisis de laboratorio: Las pruebas de laboratorio se llevaron a cabo en el Instituto de Investigaciones en Salud de la Universidad de Costa Rica. La extracción de ADN de las muestras se realizó utilizando el método convencional de extracción con fenol-cloroformo adaptado por González (2003).

Para la amplificación de los genes GSTT1 y GSTM1 se siguió el método utilizado por Lum y Le Marchand (1998) con las modificaciones de González (2003). Los iniciadores utilizados para el gen GSTT1 fueron 5'TTCCTTACTGGTCCTCACACTCT-3' y 5'TCACCGGATCATGGCCAGCA-3', y para el gen GSTM1 los iniciadores 5'-GAACTCCCTGAAAAGCTAAAGC-3' y 5'-GTTGGGGTCAAATATACGGTGG-3'.

Se coamplificó un fragmento del gen de $\beta$ globina de 268 pares de bases como control 
interno, cuyos iniciadores son 5'-CAACTTCATCCACGTTCACC-3' y 5'-GAAGAGCCAAGGACAGGTAC-3'.

Los fragmentos para los genes GSTT1 y GSTM1 corresponden a bandas de 720 pares de bases y 215 pares de bases respectivamente. En los casos donde el producto de la reacción es el polimorfismo mutado que carece de expresión enzimática (GSTT1*0 o GSTM1*0), no se observa la banda correspondiente.

Para el análisis del polimorfismo CYP1A1, alelo MspI, se siguió el protocolo utilizado por Lum y Le Marchand (1998) con las modificaciones de González (2003). Los iniciadores utilizados fueron 5'-TAGGAGTCTTGTCTCATGCCT-3' y 5-'CAGTGAAGAGGTGTAGCCGCT-3'. Del producto obtenido a partir de la PCR se tomaron $10 \quad 1$ para la reacción de digestión con $10 \mathrm{U}$ de la enzima de restricción MspI. El ADN del alelo mutante CYP1A1 MspI produjo bandas de 200 y 140 pares de bases y el alelo del gen CYP1A1 que no produjo corte por la enzima de restricción, corresponde a un fragmento de 340 pares de bases.

Finalmente para el análisis del polimorfismo genético CYP2E1 PstI se siguió el método utilizado por Anwar et al. (1996), con algunas variaciones utilizadas por González (2003). Los iniciadores utilizados para amplificar este gen fueron el 5'-CCAGTCGAGTCTACATTGTCA-3' y 5'-TTCATTCTGTCTTCTAACTGG-3'. Posteriormente $10 \mu \mathrm{l}$ del producto de la PCR fueron digeridos durante toda la noche a $37^{\circ} \mathrm{C}$ con $10 \mathrm{U}$ de la enzima de restricción PstI. La presencia de un sitio de corte para la enzima corresponde al alelo mutante (CYP2E1 PstI), en el cual se observan dos fragmentos, uno de 120 pares de bases y otro de 290 pares de bases; mientras que el alelo CYP2E1 sin sitio de corte, presenta un único fragmento de 410 pares de bases.

Análisis estadístico: Los análisis estadísticos de los datos se llevaron a cabo utilizando el programa STATA 6.0 (Stata Corp. 1999. Statistics/ Data Analysis. Texas, USA).

Para estimar diferencias entre las frecuencias de los genotipos entre los tres grupos de estudio se utilizó la prueba de $\chi^{2}$. La relación entre el cáncer gástrico y cada uno de los factores de riesgo se midió utilizando la razón de riesgo (odds ratios $=$ OR) y sus intervalos de confianza (IC) al 95\%, derivados de un análisis de regresión logística ordinal (Lindsey 1997).

\section{RESULTADOS}

La distribución según sexo y edad se puede observar en el cuadro 1. El promedio de la edad de los individuos con cáncer gástrico (60.71 años), es mayor que el promedio de edad de los individuos de los otros grupos. Del total de 140 individuos, 86 eran hombres y 54 mujeres. En el grupo de individuos con cáncer, se presentó un mayor número de hombres con la patología que de mujeres ( 25 y 6 respectivamente).

El cuadro 2 muestra la distribución de las frecuencias genotípicas, para los genes GSTM1, GSTT1, CYP1A1 y CYP2E1 en la población de estudio. No se encontraron diferencias estadísticamente significativas en las frecuencias genotípicas para ninguno de los genes, entre los tres grupos de estudio (GSTM1 p=0.89, GSTT1 p=0.88, CYP1A1 $\mathrm{p}=0.73$, CYP2E1 p=0.18).

No se encontró evidencia significativa de interacción entre el polimorfismo CYP1A1 MspI y el riesgo de cáncer de estómago o lesiones gástricas. Del mismo modo, tampoco se encontró evidencia de asociación para los diferentes genotipos de los genes GSTs (GSTT1 y GSTM1). Sin embargo, los individuos con un genotipo homocigoto del polimorfismo CYP2E1 PstI presentaron menor frecuencia de cáncer gástrico. Este alelo podría actuar como factor de protección contra el cáncer gástrico $\left(\mathrm{OR}=0.09\right.$ ajustados por edad y sexo, $\mathrm{IC}_{95 \%}$ : $0.01-0.83$ ) (cuadro 3).

\section{DISCUSIÓN}

Los homocigotos para alelo GSTM1*0, presentaron una frecuencia de $51.43 \%$ del total de la población en estudio. En términos generales se estima que las frecuencias varían entre 


\section{CUADRO 1 \\ Distribución por sexo y edad de la población de estudio $(N=140)$}

TABLE 1

Distribution by sex and age $(N=140)$

\begin{tabular}{|c|c|c|c|c|}
\hline Variable & Sanos n (\%) & Lesiones n (\%) & Cáncer n (\%) & Total \\
\hline \multicolumn{5}{|l|}{ Edad } \\
\hline$<48$ años & $15(29.51)$ & $16(27.59)$ & $3(9.68)$ & $34(24.29)$ \\
\hline 48-58 años & $15(29.41)$ & $14(24.14)$ & $8(25.81)$ & $37(26.43)$ \\
\hline 59-66 años & $12(23.00)$ & $12(20.69)$ & $11(35.48)$ & $35(25.00)$ \\
\hline$>66$ años & $9(17.65)$ & $16(27.59)$ & $9(29.03)$ & $34(24.29)$ \\
\hline Promedio de edad & $51.73 \pm 16.54$ & $55.69 \pm 15.34$ & $60.71 \pm 10.67$ & \\
\hline Ámbito de edad & $18-76$ & $23-93$ & $32-78$ & \\
\hline \multicolumn{5}{|l|}{ Sexo* } \\
\hline Femenino & $18(35.29)$ & $30(51.72)$ & $6(19.35)$ & $54(38.57)$ \\
\hline Masculino & $33(64.72)$ & $28(48.28)$ & $25(80.65)^{* *}$ & $86(61.43)$ \\
\hline
\end{tabular}

\section{CUADRO 2}

Frecuencias genotípicas de los genes GSTM1, GSTT1, CYP1A1 y CYP2E1 en cada uno de los grupos de estudio

TABLE 2

GSTM1, GSTT1, CYP1A1 and CYP2E1 genotypic frequency by group

\begin{tabular}{|c|c|c|c|c|}
\hline Genotipo & Casos $(\%)$ & $\begin{array}{c}\text { Grupo } \\
\text { Grupo con lesión }(\%)\end{array}$ & Controles (\%) & Número de individuos (\%) \\
\hline GSTM1 & 31 & 58 & 51 & \\
\hline GSTM $1 * 0 / \mathrm{GSTM} 1 * 0$ & $15(48.39)$ & $31(53.45)$ & $26(50.98)$ & $72(51.43)$ \\
\hline GSTM1/GSTM1 y GSTM1/GSTM1*0 & $16(51.61)$ & $27(46.55)$ & $25(49.02)$ & $68(48.57)$ \\
\hline $\mathrm{p}$ & 0.89 & & & \\
\hline GSTT1 & 31 & 58 & 51 & \\
\hline GSTT $1 * 0 / \mathrm{GSTT} 1 * 0$ & $6(19.35)$ & $11(18.97)$ & $8(15.69)$ & $25(17.86)$ \\
\hline GSTT1/GSTT1 y GSTT1*0/GSTT1 & $25(80.65)$ & $47(81.03)$ & $43(84.31)$ & $115(82.14)$ \\
\hline $\mathrm{p}$ & 0.88 & & & \\
\hline CYP1A1 & 31 & 58 & 51 & \\
\hline CYP1A1/ CYP1A1 & $10(32.26)$ & $25(43.10)$ & $22(43.14)$ & $57(40.71)$ \\
\hline CYP1A1/ CYP1A1 Mspl & 19(61.29) & $27(46.55)$ & $25(49.02)$ & $71(50.71)$ \\
\hline CYP1A1 MspI /CYP1A1 Mspl & $2(6.45)$ & $6(10.34)$ & $4(7.84)$ & $12(8.57)$ \\
\hline $\mathrm{p}$ & 0.73 & & & \\
\hline CYP2E1 & 31 & 58 & 51 & \\
\hline CYP2E1/ CYP2E1 & $20(65.52)$ & $39(67.24)$ & $31(60.78)$ & $90(64.29)$ \\
\hline CYP2E1/CYP2E1 PstI & $11(35.48)$ & $18(31.03)$ & $15(29.41)$ & $44(31.43)$ \\
\hline CYP2E1 PstI/ CYP2E1 PstI & $0(0.00)$ & $1(1.72)$ & $5(9.80)$ & $6(4.29)$ \\
\hline $\mathrm{p}$ & 0.18 & & & \\
\hline
\end{tabular}


CUADRO 3

Asociación entre el polimorfismo CYP2E1 PstI y el riesgo de cáncer gástrico

TABLE 3

Association between CYP2E1 PstI polymorphism and gastric cancer risk

Genotipo

CYP2E1/CYP2E1

CYP2E1 PstI/CYP2E1

CYP2E1 PstI/CYP2E1PstI

* ajustados por edad y sexo

Casos $\quad \begin{gathered}\text { Grupo con } \\ \text { lesión }\end{gathered}$

20

11

0
Controles

normales

39

18

1
31

15

5
Odds ratio*
$\quad(95 \%)$$$
1
$$$$
0.95
$$

0.09
Intervalo de confianza (95\%)

$\begin{array}{ll}0.53-2.10 & 0.88 \\ 0.01-0.83 & 0.03\end{array}$

$\mathrm{p}$

.03 un 30 a 70\%, en diferentes poblaciones (ElZein et al. 1997, Setiawan et al. 2000).

Los individuos con el genotipo homocigota para el alelo nulo del gen GSTM1, no presentaron diferencia en el riesgo de padecer de cáncer gástrico o lesiones precancerosas con respecto a los individuos que poseían el alelo normal. Este mismo resultado se encontró en otros estudios (Deakin et al. 1996, Kato et al. 1997, Setiawan et al. 2000, Lan et al. 2001, Wu et al. 2002). Sin embargo, otras investigaciones han obtenido una asociación positiva entre el riesgo de cáncer gástrico y la presencia de este alelo. Cai et al. (2001) observaron un exceso de homocigotos para el alelo GSTM $1 * 0$ en los pacientes con cáncer gástrico $\left(\mathrm{OR}=2.63 \mathrm{IC}_{95 \%}: 1.17-5.88\right)$, y a la vez, en los individuos fumadores portadores de este genotipo un riesgo mucho mayor $\left(\mathrm{OR}=8.06 \mathrm{IC}_{95 \%}\right.$ : 2.83-23.67). Saadat y Saadat (2001) reportaron un incremento en el riesgo de cáncer gástrico de 2.3 veces en los pacientes homocigotas para dicho genotipo. Otros investigadores como Harada et al. (1992) también encontraron un mayor riesgo en los portadores de este genotipo. Katoh et al. (1996) encontraron una débil asociación entre los individuos con el polimorfismo nulo y el riesgo de cáncer gástrico.

Los homocigotos para el alelo GSTT $1 * 0$, representaron el $17.86 \%$ de la población de estudio. Otros investigadores han reportado la frecuencia de este genotipo entre el $10 \%$ y hasta el $65 \%$ de los individuos en varias poblaciones (Setiawan et al. 2000). El alelo nulo para el gen GSTT1, del mismo modo que el alelo nulo para el gen GSTM1, tampoco mostró asociación con el riesgo de desarrollo del cáncer gástrico. Otros investigadores obtuvieron un resultado similar (Deakin et al. 1996, Katoh et al. 1996, Cai et al. 2001, Saadat y Saadat 2001, Wu et al. 2002). Sin embargo, Setiawan et al. (2000) y Lan et al. (2001) difieren con estos resultados, ya que encontraron un riesgo mayor de cáncer gástrico en individuos homocigotas para el genotipo nulo $(\mathrm{OR}=1.48$ $\mathrm{IC}_{95 \%}: 0.97-2.25$ y $\mathrm{OR}=2.5 \mathrm{IC}_{95 \%}: 1.01-6.22$ respectivamente).

Las proteínas de la superfamilia GST están involucradas en la desintoxicación de una amplia variedad de compuestos potencialmente cancerígenos (Lan et al. 2001). Aún no está claro cuales de las sustancias que metabolizan estas proteínas están relacionadas con un incremento en el riesgo del cáncer de estómago. Por esta razón, son necesarios más estudios que confirmen la asociación de los genotipos nulos del gen GSTT1 y el riesgo de cáncer de estómago con el fin de elucidar el papel de este polimorfismo en la patogénesis de este mal.

Para el gen CYP1A1, $8.57 \%$ de individuos presentaron el genotipo homocigota del alelo $M s p I$ y $50.71 \%$ fueron heterocigotas para dicho alelo. Este valor es un poco mayor a los observados en otras etnias, en las cuales la frecuencia reportada de los homocigotos para el alelo MspI varía entre 0.5 a 5\% (D'Errico et al. 1999). No se encontró ninguna asociación 
entre el cáncer gástrico y los diferentes genotipos del gen CYP1A1. Este polimorfismo ha sido poco estudiado como factor de riesgo del cáncer gástrico y en ninguna investigación hasta la fecha se ha encontrado algún tipo de asociación. En dos estudios no se encontró diferencias en la distribución de las frecuencias de los genotipos MspI o Ile/ $\mathrm{Val}$ entre los pacientes con cáncer de estómago y los controles (Kawajiri et al. 1993, Oda et al. 2000). Tatemichi et al. (1999), reportaron la expresión de CYP1A1 y CYP1A2 en muestras de metaplasia intestinal y concluyeron que al parecer su expresión en la mucosa gástrica es alta en las células cancerosas, por lo cual la proteína podría contribuir en la transformación de sustancias en mutágenos, aumentando la susceptibilidad del individuo. Por ello, este polimorfismo es interesante y debe ser estudiado más a fondo en su relación con este tipo de cáncer. Este polimorfismo podría estar involucrado en el desarrollo más rápido del tumor, transformando una mayor cantidad de sustancias en carcinogénos.

La frecuencia de los homocigotos con el polimorfismo PstI del gen CYP2E1 fue baja. Se observó una frecuencia de homocigotos de $4.29 \%$ y de los heterocigotas de $31.43 \%$. Este resultado es similar al de otras poblaciones en las cuales la frecuencia de los homocigotos para este polimorfismo varía entre un 2 a un $8 \%$ (Kato et al. 1996, El-Zein et al. 1997 y Nishimoto et al. 2000). Sin embargo, las frecuencias en asiáticos son mucho mayores (alrededor de $47 \%$ de los casos y $48 \%$ de los controles en un estudio caso-control) (Nishimoto et al. 2000).

Este estudio sugiere un efecto protector contra el cáncer gástrico conferido por el genotipo homocigoto para el polimorfismo CYP2E1 PstI. Otros investigadores han reportado resultados similares, tal es el caso de Nishimoto et al. (2000), quienes encontraron un riesgo menor asociado al polimorfismo RsaI (posiblemente la misma mutación que $P s t I$ ) en una población de brasileños no japoneses y en una población de brasileños de origen japonés. Oda et al. (2000) y Marchant et al. (1999), también encontraron asociación entre este alelo y una disminución en el riesgo de este tipo de cáncer.
Si esto es confirmado quedaría abierta la posibilidad de que otras variables enzimáticas de este tipo, pudieran modular el riesgo del desarrollo del cáncer gástrico (González et al. 2002).

Otras investigaciones han obtenido resultados diferentes. Gao et al. (2002), Kato et al. 1995, Kato et al. 1996, Kato et a.l 1997), no encontraron ningún tipo de asociación entre este polimorfismo y el riesgo de cáncer gástrico. Aunque, Gao et al. (2002) encontraron que los individuos homocigotas y heterocigotas con este polimorfismo presentan un incremento en el riesgo cuando fuman y consumen altas cantidades de carne. Wu et al. (2002), encontraron una asociación positiva significativa entre las personas homocigotas para el alelo PstI con un riesgo 2.9 veces mayor de padecer cáncer gástrico $\left(\mathrm{IC}_{95 \%}\right.$ : 1.4-6.4). Ellos sugieren que este hallazgo es compatible con el hecho de que esta proteína cataliza la oxidación de varias sustancias, especialmente de procarcinógenos de bajo peso molecular como las nitrosaminas, a carcinógenos capaces de formar aductos en el ADN (Wu et al. 2002).

Es evidente que para lograr una interpretación más adecuada de este tipo de estudios falta una mayor investigación ya que el cáncer es multifactorial. Se deben conocer más las interacciones genes-ambiente, incluyendo la dieta y el fumado, ya que los factores ambientales alteran la actividad enzimática y modifican la susceptibilidad del riesgo al cáncer (Gao et al. 2002). De hecho, en diferentes investigaciones se han reportado diferencias en la actividad de la enzima CYP2E1 por etnia y género. Se estima que existe una actividad enzimática menor en un $25 \%$ para las mujeres y que la actividad en los japoneses es entre 30 a $40 \%$ menor que en los caucásicos (O'Shea et al. 1994, Kim et al. 1995, Kim et al. 1996).

La disminución en el riesgo del desarrollo del cáncer gástrico en los pacientes con el alelo PstI o RsaI observado en ésta y otras investigaciones, podría deberse a que este polimorfismo presenta una mayor actividad transcripcional y a que participa en el metabolismo de los compuestos N-nitrosos. Según Hayashi et al. 
(1991), el incremento en la expresión de este polimorfismo en los homocigotos es diez veces mayor. Sin embargo, se desconoce con exactitud el mecanismo a través del cual este polimorfismo podría reducir el riesgo (Marchant et al. 1999, Gao et al. 2002). La enzima de CYP2E1 metaboliza una gran cantidad de sustancias de diferentes estructuras moleculares, lo cual podría indicar que no presenta una afinidad alta por el sustrato, por lo que en ciertos casos se necesitaría gran cantidad de sustrato para que se realice la desintoxicación (Guengerich et al.1991, citado por Lang y Pelkonen 1999). De este modo, es posible que el genotipo CYP2E1 Pst1, represente protección contra el cáncer gástrico por poseer una mayor actividad transcripcional y por lo tanto una mayor capacidad para desintoxicar sustancias.

La regulación de la enzima CYP2E1 es compleja e involucra eventos tanto a nivel transcripcional como a nivel postranscripcional, lo que hace difícil demostrar su asociación con el cáncer (Eliasson et al. 1992). Es importante aclarar que una variable alélica para un gen del metabolismo de los xenobióticos, podría causar efectos dañinos en una población con exposición alta a un determinado carcinógeno ambiental. No obstante, en la ausencia de los carcinógenos, un individuo portador del alelo de alto riesgo podría no presentar ningún efecto en el riesgo de cáncer gástrico (González et al. 2002).

Es importante tener en cuenta que los rangos de edades de los individuos seleccionados para este estudio es amplio. Existe la posibilidad de que algunas de las personas más jóvenes que no presentan cáncer gástrico llegaran a desarrollarlo en el futuro ya que la edad es un importante factor de riesgo de cáncer gástrico tipo intestinal.

Casi todos los estudios, al igual que esta investigación, se basan en pruebas de genotipos asociados a la enfermedad, sin embargo el fenotipo de las enzimas de desintoxicación química es un fenómeno muy complejo, en el cual múltiples y desconocidos factores entran en juego. Tanto factores endógenos como exógenos podrían inducir o inhibir la actividad enzimática (estado hormonal, estrés oxidativo, edad y enfermedades que haya padecido el individuo, interacciones droga-droga, hábitos alimenticios). Por esta razón debe estudiarse más profundamente la relación entre el genotipo-fenotipo, en el caso de estos genes con el fin de interpretar de una mejor manera los resultados obtenidos en este tipo de estudios (Vineis y Malats 1999).

Como muestran los resultados de esta investigación, no se encontró ningún tipo de asociación estadísticamente significativa entre los genotipos nulos de los genes GSTT1 y GSTM1 ni con el polimorfismo CYP1A1 MspI con el riesgo de desarrollar cáncer gástrico; pero si se encontró una asociación significativa entre la presencia del polimorfismo CYP2E1 Pst1 y una disminución del riesgo de padecer cáncer gástrico. Con una mayor investigación y junto a los resultados obtenidos en otras investigaciones, en un futuro se tendrá mayor claridad respecto a la susceptibilidad proporcionada por estos genes como factores de riesgo de cáncer gástrico.

\section{AGRADECIMIENTOS}

Un sincero agradecimiento al personal del INISA, al CONICIT, a Jorge Lobo por el apoyo estadístico y al Sistema de Estudios de Posgrado en Biología de la Universidad de Costa Rica. Esta investigación fue financiada por la Vicerrectoría de Investigación de la Universidad de Costa Rica (proyecto N.742-AO-025).

\section{RESUMEN}

Las enzimas de las familias P450 y Glutation Stransferasa están relacionadas en la activación y desintoxicación de sustancias que podrían actuar como cancerígenas. Polimorfismos genéticos en estas enzimas han sido asociados con un incremento en el riesgo de desarrollar cáncer específicamente con un mayor riesgo de desarrollar cáncer gástrico. En esta investigación se estudió un grupo de costarricenses con alto riesgo de cáncer gástrico. Se estudiaron 31 individuos con cáncer gástrico, 51 controles normales confirmados por rayos X (serie gastroduenal de 
doble contraste) o por endoscopía y 58 individuos con otras lesiones gástricas. Se estudiaron los polimorfismos de desintoxicación química CYP1A1 MspI y CYP2E1 Ps$t I$, los cuales presentan una mayor expresión enzimática y los polimorfismos de los genes GSTT1 y GSTM1 que carecen de un producto proteínico funcional y su relación con lesiones gástricas leves y cáncer gástrico. El ADN de los pacientes fue aislado a partir de leucocitos de sangre periférica. Los polimorfismos de los genes GSTT1 y GSTM1 fueron evaluados mediante un PCR múltiple y para los polimorfismos CYP2E1 PstI y CYP1A1 MspI se realizó un PCR seguido por la digestión con las enzimas de restricción PstI y MspI respectivamente. La prevalencia del polimorfismo CYP1A1 MspI, y de los polimorfismos GSTT1 y GSTM1 sin actividad enzimática, fue similar en los tres grupos estudiados $(\mathrm{p}=0.73, \mathrm{p}=0.88 \mathrm{y} \mathrm{p}=0.89$ respectivamente). Los resultados sugieren que el alelo CYP2E1 Pst1 podría actuar como factor protector contra el cáncer gástrico $(\mathrm{OR}=0.09, \mathrm{IC}$ al 95\%: 0.01-0.83).

\section{REFERENCIAS}

Anónimo. 1997. Stomach. Food, Nutrition and the prevention of cancer: a global perspective. World Cancer Research. American Institute for Cancer Research. pp. 148-175.

Anwar, W., S. Abdel-Rahman, R. El-Zein, H. Mostafa \& W. Au. 1996. Genetic polymorphism of GSTM1, CYP2E1 and CYP2D6 in Egyptian bladder cancer patients. Carcinogenesis 17: 1923-1929.

Cai, L., S. Yu \& Z. Zhang. 2001. Gluathione S-transferases M1, T1 genotypes and the risk of gastric cancer: A case-control study. World J. Gastroenterol. 7: 506-509.

Correa, P. 1992. Human gastric carcinogenesis: A multistep and multifactorial process. Cancer Res. 52: 673566740 .

Deakin, M., J. Elder, C. Hendrickse, D. Peckham, D. Baldwin, C. Pantin, N. Wild, P. Leopard, D. Bell, P. Jones, H. Duncan, K. Brannigan, J. Alldersea, A. Fryer \& R. Strange. 1996. Glutathione S-transferase GSTT1 genotypes and susceptibility to cancer: studies of interactions with GSTM1 in lung, oral, gastric and colorectal cancers. Carcinogenesis 17: 881-884.

D’ Errico, A., N. Malats, P. Vineis \& P. Boffetta. 1999. Review of studies of selected metabolic polymorphism and cancer. pp.323-393. In P. Vineis, N. Malats, M. Lang, A. D' Errico, N. Caporaso, J. Cuzick \& P. Boffeta (eds.). Metabolic polymorphisms and susceptibility to cancer. Scientific Publications.

Eliasson, E., S. Mkrtchian \& M. Ingelman-Sundberg. 1992. Hormone- and substrate-regulated intracellular degradation of cytochrome P450 (2E1) involving $\mathrm{MgATP}-a c t i v a t e d$ rapid proteolysis in the endoplasmic reticulum membranes. J. Biol. Chem. 267: 15765-15769.

El-Omar, E., M. Carrington, W. Chow, K. Mc Coll, J. Bream \& H. Young. 2000. Interleukin-1 polymorphisms associated with increased risk of gastric cancer. Nature 404: 398-402.

El- Zein, R., N. Conforti-Froes \& W. Au. 1997. Interactions between genetic predisposition and environmental toxicants for development of lung cancer. Environ. Mol. Mutagen. 30: 192-204.

Ferlay, J., F. Bray, P. Pisani \& D. Parkin. 2001. GLOBOCAN 2000: Cancer Incidence, Mortality and Prevalence Worldwide, Version 1.0. IARC CancerBase No. 5. Lyon, IARC.

Gao, Ch., T. Takezaki, J. Wu, Z. Li, J. Wang, J. Ding, Y. Liu, X. Hu, T. Xu, K. Tajima \& H. Sugimura. 2002. Interaction between Cytochrome P450 2E1 Polymorphism and environmental factors with risk of esophageal and stomach cancer in chinese. Cancer Epidemiol. Biomarkers Prev. 11: 29-34.

González, M. 2003. Polimorfismos en los genes de desintoxicación química CYP1A1, CYP2E1, GSTT1 y GSTM1 en la susceptibilidad al cáncer gástrico. Tesis de Maestría. Universidad de Costa Rica. San José, Costa Rica. 77 p.

González, C., N. Sala \& G. Capella. 2002. Genetic susceptibility and gastric cancer risk International J. Cancer. 100: 249-260.

Harada, S., S. Misawa, T. Nakamura, N. Tanaka, E. Ueno \& O. Nyren. 1992. Delection of GSTM1 gene deletion by the polymerase chain reaction and its possible correlation with stomach cancer in Japanese. Human Genet. 90: 62-64.

Hayashi, S., J. Watanabe \& K. Kawajiri. 1991. Genetic polymorphisms in the 5'-flanking region change transcriptional regulation of the human cytochrome P450IIE1 gene. J. Biochem. Tokyo. 110: 559-565.

Hengstler, J., M. Arand, M. Herrero \& F. Oesch. 1998. Polimorphisms of N-acetyltransferases, glutathione Stransferases, microsomal epoxide hydrolase and sulfotransferases: influence on cancer susceptibility. Recent Results Cancer Res. 154: 47-85.

Kato, S., M. Onda, N. Matsukura, A. Tokunaga, N. Matsuda, K. Yamashita \& P. Shields. 1997. Helicobacter pylori infection and genetic polymorphisms for cancer-related in gastric carcinogenesis. Biomed. Pharmacother. 51: 145-149. 
Kato, S., M. Onda, N. Matsukura, A. Tokunaga, N. Matsuda, S. Yamashita \& P. Shields. 1996. Genetic polymorphism of the cancer related gene and Helicobacter pylori infection in Japanese gastric cancer patients. An age and gender matched case-control study. Cancer 77: 1654-1661.

Kato, S., M. Onda, N. Matsukura, A. Tokunaga, T. Tajiri, D. Kim, H. Tsuruta, N. Matsuda, S. Yamashita \& P. Shields. 1995. Cytochrome P450 (CYP2E1) genetic polymorphism in a case-control study of gastric cancer and liver disease. Pharmacogenetics 5: S141S144.

Katoh, T., Y. Kuroda, H. Itoh, A. Kawahara, N. Kuroki, R. Ookuma \& D.A. Bell. 1997. Gluthatione S-transferase M1 (GSTM1) and T1 (GSTT1) genetic polymorphism and susceptibility to gasttric and colorectal adenocarcinoma. Carcinigenesis 17: 1855-1859.

Kawajiri, K. 1999. CYP1A1. pp. 159-172. In P. Vineis, N. Malats, M. Lang, A. D' Errico, N. Caporaso, J. Cuzick \& P. Boffeta (eds.). Metabolic polymorphisms and susceptibility to cancer. Scientific Publications.

Kawajiri, K., K. Nakachi, K. Imai, J. Watanabe \& S. Hayashi. 1993. The CYP1A1 gene and cancer susceptibility. Critical Reviews In Oncology/Hematology. 14: 77-87.

Kim, R., H. Yamazaki, K. Chiba, D. O’Shea, M. Mimura, F. Guengerich, T. Ishizaki, T. Shimada \& G. Wilkinson. 1996. In vivo and in vitro characterization of CYP2E1 activity in Japanese and Caucasians. J. Pharmacol. Exp. Therapeut. 279: 4-11.

Kim, R. \& D. O’Shea. 1995. Interindividual variability of chlorzoxazone 6-hydroxylation in men and women and its relationship to CYP2E1 genetic polymorphisms. Clin. Pharmacol. Therapeut. 57: 645-655.

Krajinovic, M., D. Labuda, C. Richer, S. Karimi \& D. Sinnett. 1999. Susceptibility to Childhood acute Lymphoblastic Leukemia: Influence of CYP1A1, CYP2D6, GSTM1 and GSTT1 Genetic Polymorphisms. Blood 93: 1496-1501.

Lan, Q., W. Chow, J. Lissowska, D. Hein, K. Buetow, L. Engel, B. Ji, W. Zatonski \& N. Rothman. 2001. Glutathione S-transferase genotypes and stomach cancer in a population-based case-control study in Warsaw, Poland. Pharmacogenetics 11: 655-661.

Lang, M. \& O. Pelkonen. 1999. Metabolism of xenobiotics and chemical carcinogenesis. pp. 13-22. In P. Vineis, N. Malats, M. Lang, A. D’ Errico, N. Caporaso, J. Cuzick \& P. Boffeta (eds.). Metabolic polymorphisms and susceptibility to cancer. Scientific Publications.
Lindsey, J. 1997. Applying Generalized Linear Models. Springer-Verlag. New York. 256 p.

Lum, A. \& L. Le Marchand. 1998. A simple Mouthwash for obtaining genomic DNA in molecular epidemiological studies. Cancer Epidemiol. Biomarkers Prev. 7: 719-724.

Marchant, L., G. Wilkinson \& L. Wilkens. 1999. Genetics and dietary predictors of CYP2E1 activity: A phenotyping study in Hawaii Japanese using cholorzoxazone. Cancer Epidemiol. Biomarkers Prev. 8: 495500 .

Nishimoto, I., T. Hanaoka, H. Sugimura, K. Nagura, M. Ihara, X. Li, T. Arai, G. Hamada, L. Kowalski \& S. Tsugane. 2000. Cytochrome P450 2E1 polymorphism in gastric cancer in Brazil: Case-control studies of Japanese Brazilians and non-Japanese Brazilians. Cancer Epidemiol. Biomarkers Prev. 9: 675-680.

O’Shea, D., S. Davis, R. Kim \& G. Wilkinson. 1994. Effect of fasting and obesity in humans on the 6-hydroxylation of chlorzoxazone: a putative probe of CYP2E1 activity. Clin. Pharmacol. Therapeut. 56: 359-367.

Oda, Y., S. Suzuki \& I. Nakanishi. 2000. Genotypes of cytochromes P450 1A1 and 2E1 in Japanese Patients with gastric cancer. Pathol. Int. 50: Suplem: A92.

Parkin, M. \& P. Pisani. 1999. Gastric Cancer. pp. 515-529. In B. Kramer, J. Gohagan \& P. Prorok (eds.). Cancer screening. Theory and practice. Marcel Dekker, New York.

Saadat, I. \& M. Saadat. 2001. Glutathione S-transferase M1 and T1 null genotypes and the risk of gastric and colorectal cancers. Cancer Letters 169: 21-26.

Setiawan, V., Z. Zhang, G. Yu, Y. Li, M. Lu, C. Tsai, D. Cordova, M. Wang, C. Guo, S. Yu \& R. Kurtz. 2000. GSTT1 and GSTM1 null genotypes and the risk of gastric cancer. A case control study in a Chinese population. Cancer Epidemiol. Biomarkers Prev. 9: 73-80.

Shibata, A., T. Longacre, B. Puligandla, J. Parsonnet \& A. Habel. 2001. Histological classification of gastric adenocarcinoma for epidemiological research: concordance between pathologists. Cancer Epidemiol. Biomarkers Prev. 10: 75-78.

Sierra, R. 2002. Cáncer gástrico, epidemiología y prevención. Acta Méd. Costarric. 44: 55-61.

Sierra, R., L. Rosero-Bixby, D. Antich \& G. Muñoz. 1995. Cáncer en Costa Rica: Epidemiología descriptiva: mortalidad 1970-1990, incidencia 1984-1990. Primera edición. Editorial de Costa Rica. San José. Costa Rica. 87 p. 
Tatemichi, M., S. Nomura, T. Ogura, H. Sone, H. Nagata \& H. Esumi. 1999. Mutagenic activation of environmental carcinogens by microsomes of gastric mucosa with intestinal metaplasia. Cancer Res. 59: 3893-3898.

Vineis, P. \& N. Malats. 1999. Strategic issues in the design and interpretation of studies on metabolic polymorphism and cancer. pp. 51-61. In P. Vineis, N. Malats,
M. Lang, A. D’ Errico, N. Caporaso, J. Cuzick \& P. Boffeta (eds.). Metabolic polymorphisms and susceptibility to cancer. Scientific Publications.

Wu, M-S., Ch-J. Chen, M-T. Lin, H-P. Wang, C-T, Shun, J-C. Sheu \& J-T. Lin. 2002. Genetic polymorphisms of cytochrome p450 2E1, Glutathione S-transferase M1 and T1, and susceptibility to gastric carcinoma in Taiwan. Int. J. Colorectal Dis. 17: 338-343. 